\title{
Optimized workflow for digitalized FISH analysis in pathology
}

\author{
Vira Chea ${ }^{1}$, Valerie Pleiner ${ }^{1}$, Viviane Schweizer ${ }^{1}$, Benjamin Herzog ${ }^{2}$, Beata Bode ${ }^{1}$ and Marianne Tinguely ${ }^{{ }^{*}}$ (D)
}

\begin{abstract}
Background: Effective workflow management in a diagnostic pathology laboratory is critical to achieve rapid turnover while maintaining high quality. Fluorescence in situ hybridization analysis (FISH) is the preferred technique for detecting single chromosomal aberrations in diagnostic surgical pathology.

Material and methods: FISH analysis applying a rapid hybridization protocol and using an automated whole-slide fluorescence scanning device (3DHISTECH, Sysmex, Switzerland) were implemented in our workflow. By analyzing 42 diagnostic cases, effects of two different scanning profiles on scanning time, and device memory usage were investigated. Manual signal counting (CaseViewer) and software based signal counting (FISHQuant) were compared.

Results: The two scanning profiles, both including a Z-stack function, differed in their exposure time and digital gain. The "low profile" setting (LP) resulted in a significantly shorter scanning time and lower storage volume compared to the "high profile" (HP) setting, making the LP ideal for routine applications. Both signal counting methods (manual versus software based) provided similar cut-offs on a test-cohort of 13 samples.

Conclusion: Scanning FISH slides provides good picture quality, reduces the analysis time and allows easy picture archiving and facilitates remote diagnostics, allowing an effective workflow.
\end{abstract}

Keywords: Digital pathology, Image analysis, FISH, Lymphoma, Sarcoma

\section{Introduction}

Interphase fluorescence in situ hybridization (FISH) has gained importance as diagnostic and predictive examination in pathology [1]. Together with its cost effectiveness, it allows for a rapid target-oriented analysis providing results within a day.

In most instances, FISH slides are analyzed by an epifluorescent microscope, with or without a motorized scanning platform. Signal counting is done either manually at the microscope or at a computer screen or automatically by software-supported algorithms.

Bright field whole-slide imaging (WSI) for Hematoxylin and Eosin (H\&E) stained slides and immunohistochemistry is already used in many routine diagnostic laboratories.

\footnotetext{
* Correspondence: marianne.tinguely@patho.ch

${ }^{1}$ Institute of Pathology Enge, Hardturmstr. 133, CH-8055 Zurich, Switzerland Full list of author information is available at the end of the article
}

However, scanning FISH slides is not widely used yet. Our aim was to introduce the WSI for FISH slides into routine diagnostics. A further goal was to accelerate and standardize the FISH analysis process by introducing a rapid hybridization protocol and an automated cell-signal counting program.

Herein, we report our experiences in establishing an optimal workflow for our digitalized FISH technique. Furthermore, we discuss the pros and cons of different scanning profiles and the value of an automated cell counting software.

\section{Materials and methods \\ Pre-analytical workflow}

First, a representative tumor area was encircled on the H\&E-slide by a pathologist. Second, the corresponding area was marked with a diamond pen on the back of the slide to be hybridized. This narrowed down the tissue 
surface to be scanned since the diamond scratches remain visible on the scan-preview.

\section{FISH technique}

A standard protocol was established for FISH on formalin fixed, paraffin embedded (FFPE) specimens. A tissuemicro-array (TMA) with ten cores (at $3 \mathrm{~mm}^{2}$ diameter) for the probe set up and 42 diagnostic samples including core needle and excisional biopsies were analyzed. Adapted to the tissue type, the pretreatment time varied from 30 to $40 \mathrm{~min}$. The specific probes (Zytovision, Germany or Vysis, Abbott Molecular, USA) were hybridized at $37^{\circ} \mathrm{C}$ for $4 \mathrm{~h}$ in the presence of the IntelliFISH Hybridization buffer (Vysis, Abbott Molecular, USA). As nuclear stain and mounting medium the DAPI $\left(4^{\prime}, 6\right.$ diamidino-2-phenylindole) VECTASHIELD ${ }^{\circ}$ HardSet $^{\text {ix }}$ (Vector laboratories, CA, USA) with a minimum hardening time of $30 \mathrm{~min}$ was used.

\section{Slide imaging and analyzing}

The optical system of the Pannoramic 250 Flash II Scanner (3DHISTECH, Sysmex, Switzerland) contains two Zeiss (Jena, Germany) Plan-Apochromat dry objectives $(20 \mathrm{x}$ and $40 \mathrm{x}$ with a numerical aperture of 0.80 and 0.95 respectively) allowing also bright field scanning. In a motorized software-controlled wheel, three fluorescence filters are incorporated: FITC (green light, 459 $\mathrm{nm}$ ), TRITC (red light, $544 \mathrm{~nm}$ ) and DAPI (autofluorescence, $360 \mathrm{~nm}$ ). The "SPECTRA light (Lumencor, USA) engine 6" switches the filters fast without fading. Images are acquired by the 16-bit scientific CMOS pco.edge 4.2 camera.

We scanned all slides with the 40x objective with a resolution of $0.25 \mathrm{um} /$ pixel. To circumvent inherent tissue-quality fluctuations, two main scanning profiles (low (LP) and high (HP)) were generated. The profiles differ in their exposure time (ET) (LP: $150 \mathrm{~ms}$ vs HP: $2000 \mathrm{~ms}$ ) for the FITC and TRITC channels and their digital gain (LP: 3-4 vs HP: 0-2). For both profiles, the Z-stack function was activated using five to seven layers with a layer distance of $0.4 \mu \mathrm{m}$. The scanning time $(\mathrm{min})$, the file size $(\mathrm{MB})$ and the fields of view (FOV) of the two profiles were compared (Table 1B). The areascanning technology of the current scanner is FOV based. The FOV corresponds to the square image of the camera sensor. The larger the area to be scanned, the greater the number of the FOV required. When using different profiles with the identical area to be scanned, the number of FOV remains the same.

\section{Manual counting}

Digitalized images were visualized in the CaseViewer (3DHISTEC, Sysmex, Switzerland), a digital microscope application software. As a control step, the pre- selected areas of the corresponding H\&E- and FISHslides were viewed in parallel. Thereafter, FISH signals of a hundred of nuclei were counted manually at the computer screen. Cut-off levels were assessed as described earlier [2]. A signal was counted as abnormal, when the green and the red signal were two diameters of one signal apart.

\section{Software counting}

FISHQuant (3DHISTECH, Sysmex, Switzerland), is an IVD approved module allowing to automatically quantify structural and numerical FISH signal abnormalities in solid tumors and neoplasias of the hematopoietic system. Since automated classification is error prone due to tissue inherent artefacts like overlapping of nuclei, manual editing is mandatory before signing out final reports.

\section{Results \\ FISH technique}

Introducing the IntelliFISH Hybridization buffer substantially shortened the hybridization process from 18 to $4 \mathrm{~h}$ and resulted in good signal to noise ratios with strong and distinct signals (Figs. 1 and 2). The DAPI hardening mounting media VECTASHIELD ${ }^{\circ}$ HardSet $^{\text {ma }}$ proved to be the fastest option of the several types of media tested.

\section{Slide imaging and analyzing}

The handling of the scanner software turned out to be intuitive and rather easy. Both established profiles (LP and HP) provided signals of good quality, however, the HP translated a better signal to noise ratio (Fig. 1). The scanning time of a TMA core $\left(3 \mathrm{~mm}^{2}\right)$ varied between 5 to $7 \mathrm{~min}$ with a LP and 15 to $20 \mathrm{~min}$ with a HP.

The scanning time for FISH depended on the FOV reflecting the size of the selected area and the exposure time (ET) per fluorescence channel. In 16/42 samples we applied an LP (150 ms ET) and in 26/42 samples a HP (2000 ms ET) (Table 1B). With this approach, the scanning time was more than ten times longer for the HP (mean $170 \mathrm{~min}$ ) than for the LP (mean $15 \mathrm{~min}$ ). Moreover, the file size was 2.5 times larger for the HP than for the LP (Table 1B) while the mean file size per FOV remained comparable for all approaches (low: 0.32; high: 0.34 ) as expected. In four cases (one with LP and three with HP) the FOV was $>10^{\prime} 000$ resulting in high data volumes (min $2390 \mathrm{MB}$, max $7620 \mathrm{MB}$, mean $4453 \mathrm{MB}$ ) leading to the longest scanning time with the LP (72 $\mathrm{min})$ and HP (949 min). Whereas the LP showed strong enough signals to be successfully analyzed in most instances, HP improved picture quality in cases with weak signals or high background (Figs. 1b and 2). 
Table 1 Summaries of FISH cut-off and scanning profile

\begin{tabular}{llll}
\hline A) Break apart probes with comparison of cut off values from the literature. ${ }^{\circ}$ dual colour dual fusion & Cut in the literature (\%) \\
Probe & Provider & Cut off (\%) & 10 \\
BCL-2 & Zytovision & 4 & 8 \\
BCL-6 & Zytovision & 4 & 1.3 \\
CCND1 & Zytovision & 4 & $5^{\circ}$ \\
COL-A1 & Zytovision & 5 & 10 \\
EWSR1 & Vysis/Abbott & 7 & NA \\
FUS & Vysis/Abbott & 3 & 9 \\
C-MYC & Vysis/Abott & 5 & 20 \\
USP-6 & Vysis/Abbott & 3 &
\end{tabular}

B) Comparison of scanning time, file size and FOV (fields of fiew) scanned with the two profiles in 42 diagnostic cases

$\begin{array}{llll}\text { Profile } & \text { All }(\boldsymbol{n}=\mathbf{4 2}) & \text { High }(\boldsymbol{n}=\mathbf{2 6}) & \text { Low }(\boldsymbol{n}=\mathbf{1 6}) \\ \text { scanning time in } \mathbf{m i n} & 1 & 3 & 1 \\ \text { Min } & 1620 & 1620 & 15 \\ \text { Max } & 87 & 159 & 11 \\ \text { Mean, Total } & 34 & 57 & 30 \\ \text { Mean, <10'000 FOV } & & & 940 \\ \text { file size } & 17 & 17 & 458 \\ \text { Min } & 7620 & 7620 & 328 \\ \text { Max } & 794 & 1129 & 120 \\ \text { Mean, total } & 468 & 607 & 10620 \\ \text { Mean, <10'000 FOV } & & & 1708 \\ \text { FOV } & 105 & 105 & 1114 \\ \text { Min } & 58830 & 58830 & 5971 \\ \text { Max } & 3840 & 2311 & \end{array}$

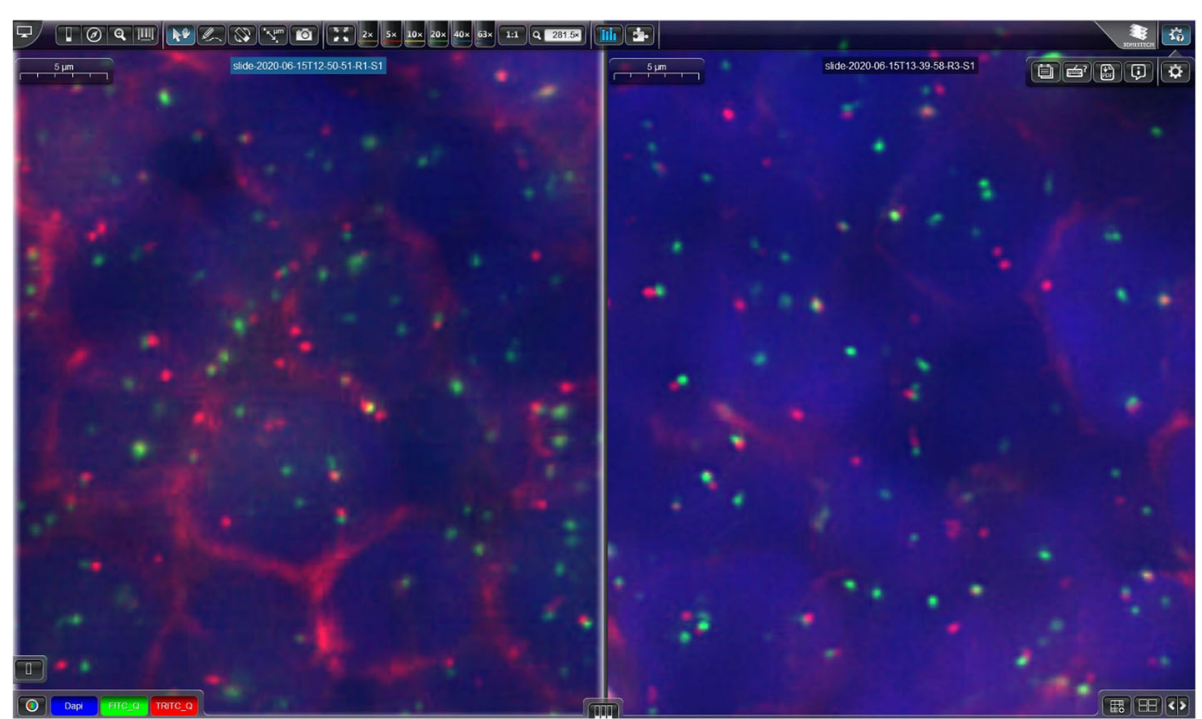

Fig. 1 Comparison of a case scanned by the low profile (a) versus high profile (b) using a C-MYC bap probe. The high profile results in a better signal to noise ratio without fading. The settings applied are shown in (c) resulting in different storage sizes 

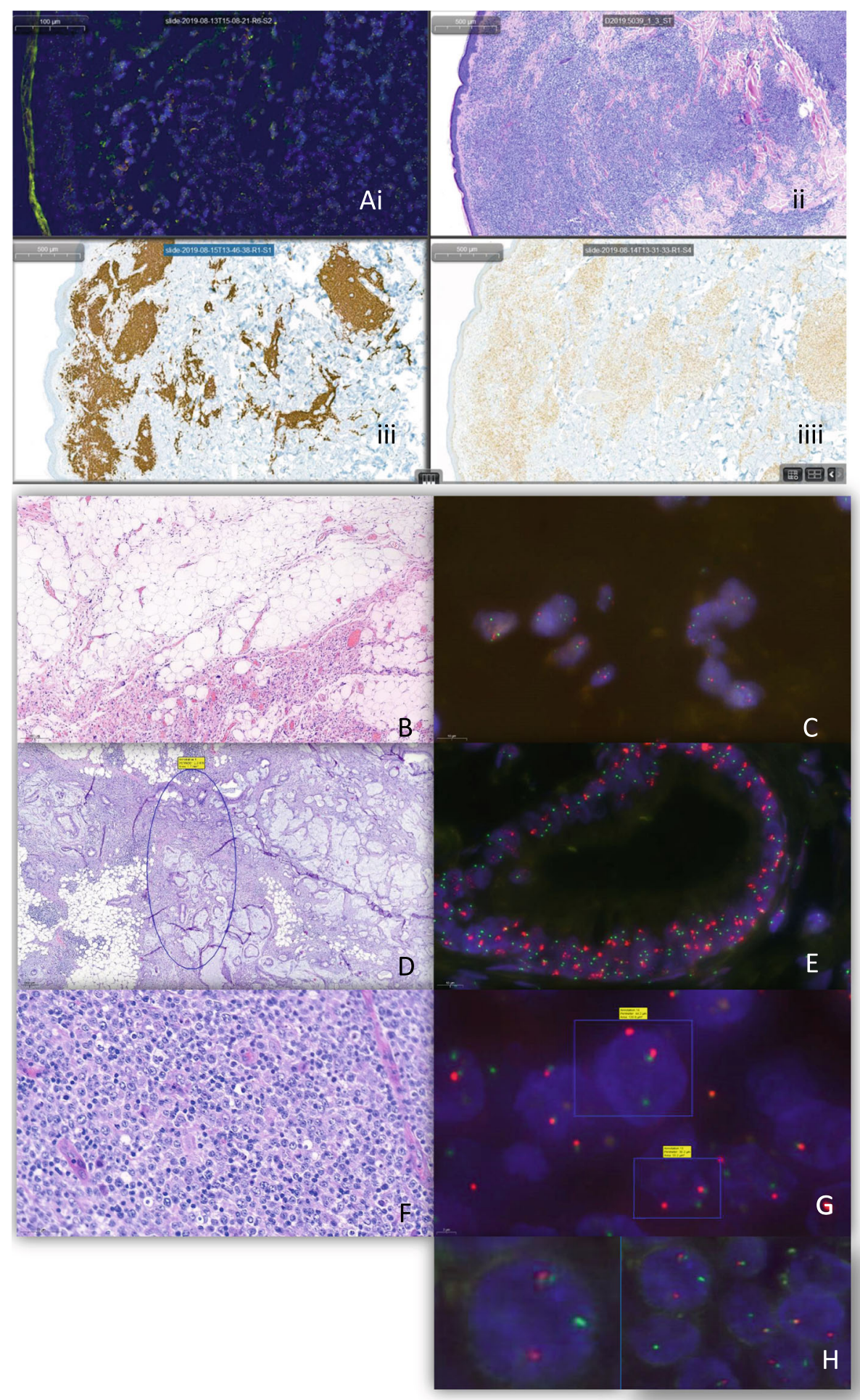

Fig. 2 Clinical samples of routine diagnostic cases with the corresponding HE: (a) Parallel viewing of a primary cutaneous marginal zone lymphoma: (i) BCL2 BAP probe, (ii) HE, (iii) CD20 (iiii) Bcl2; (b, c) Lipoma without MDM2 amplification; (d, e) Metastases of a cholangiocellular carcinoma showing the pre-analytical tissue selection area annotated on the CaseViewer and the corresponding MDM2 amplification; (f, $\mathbf{g}$ ) "double hit" lymphoma with a C-MYC break in the annotated tumour cells and a break in the BCL-6 gene (h) 


\section{Manual vs automated counting}

In three out of the 42 diagnostic samples and the TMA, the FISHQuant software automatically classified the signals and the nuclei correctly. Compared to manual counting FISHQuant provided similar results within seconds (e.g. $7 \%$ vs $6 \%$ for ETVS1). However, in the remaining samples, especially those containing lymphatic tissue, the nuclei were too densely packed to be correctly identified by the automatic algorithm, leading to a high number of erroneously classified signals.

\section{Discussion}

FISH has become an important theranostic auxiliary method in surgical pathology over the years [1]. To meet the current needs of shortening turn-around-times while maintaining high quality and cost-effectiveness, we accelerated the hybridization process by introducing the IntelliFISH hybridization buffer. Thereby, we shortened the duration of the experimental process by more than $12 \mathrm{~h}$ while preserving an excellent signal quality. The overall time from the entry of the order to the hybridized slide was cut to approximatively $6 \mathrm{~h}$ with around 30 min hands on time.

The Pannoramic 250 Flash II Scanner equipped with a fluorescent module has proven to be a reliable and efficient tool for routine diagnostics of break-apart and enumeration probes. Our observations are in line with a previous report regarding the same system and a second one dealing with a different scanning system [3, 4]. One main difference and advantage compared to conventional fluorescence microscopes is the lack of fading of the fluorescent probes during the scanning process. Additional major advantages of digitalizing FISH slides are the preview and the alignment of the hybridized slides with their corresponding H\&E or immunohistochemical stain on the CaseViewer, allowing a more precise as well as fast, identification and analysis of the diseased area (Fig. 2) [4]. Other benefits for the examiner compared to the use of a traditional fluorescence microscope were the larger fields of view and wider zoomranges. Both could be easily and continuously adjusted on the CaseViewer without losing the area of interest. This simplified analysis and the optimized FISH protocols might be reasons for the lower cut-off values for our probes as compared to those described in the literature (Table 1A) [5]. However, the methods used for the assessment of the thresholds were not indicated in all reports [6-9].

Based on our experience, the establishment of two different scanning profiles is sufficient to enable a routine diagnostic FISH laboratory to easily scan and analyze tissue samples of different origin. A mean scanning time of $15 \mathrm{~min}$ for the majority of samples applying the LP seems reasonable. Hence, the HP can be reserved for more demanding probes.

In our hands, the automated FISHQuant software is promising and provides graphically represented results of break-apart probes within seconds. However, the ability to discriminate nuclei and to correctly assign the signals to them is limited by the algorithm, necessitating an elaborate manual editing compared to the manual counting by means of the CaseViewer. Therefore, the FISHQuant software is not yet ideal for certain tissues, especially not for lymphomatous tissue, since the algorithm is only able to correctly classify a minority of nuclei. A further refinement into a self-learning system would be desirable.

In conclusion, in our view the advantages of scanning FISH slides far outweigh the conventional analysis by fluorescence microscopes. Particularly storage, sharing and remote diagnostics open up new opportunities. The development of tissue adapted self-scoring software would be desirable.

\section{Acknowledgments}

We are grateful to Patricia Braendle for excellent technical support.

\section{Authors' contributions}

VC established the protocols, analyzed the results and wrote the manuscript. VP and VS are in charge of the routine diagnostic cases for FISH and scanner. $\mathrm{BH}$ established the IT-surroundings and MT is responsible for the design and concept of the study and wrote the manuscript together with BB. All authors commented on previous versions of the manuscript. All authors read and approved the final manuscript.

\section{Funding}

The authors have not declared a specific grant for this research from any funding agency in the public, commercial or not-for-profit sectors.

\section{Declarations}

Ethics approval and consent to participate

The study was performed according to the 1964 Helsinki declaration and its amendments and Swiss Ordinance on Human Research (Art 34 HRO).

\section{Competing interests}

The authors declare no conflict of interest.

\section{Author details}

${ }^{1}$ Institute of Pathology Enge, Hardturmstr. 133, CH-8055 Zurich, Switzerland. ${ }^{2}$ County Hospital Thurgau, Frauenfeld, Switzerland.

Received: 19 February 2021 Accepted: 26 April 2021

Published online: 11 May 2021

References

1. Gupta R, Cooper WA, Selinger C, Mahar A, Anderson L, Buckland ME, et al. Fluorescent in situ hybridization in surgical pathology practice. Adv Anat Pathol. 2018;25(4):223-37. https://doi.org/10.1097/PAP.0000000000000194.

2. Curioni-Fontecedro A, Martin V, Vogetseder A, Knuth A, Moch $H$, Soldini D, et al. Chromosomal aberrations of cancer-testis antigens in myeloma patients. Hematol Oncol. 2015;33(3):159-63. https://doi.org/1 0.1002/hon.2143.

3. Laurent C, Guerin M, Frenois FX, et al. Whole-slide imaging is a robust alternative to traditional fluorescent microscopy for fluorescence in situ hybridization imaging using break-apart DNA probes. Hum Pathol. 2013; 44(8):1544-55. https://doi.org/10.1016/j.humpath.2012.12.009. 
4. Liew M, Rowe L, Clement PW, Miles RR, Salama ME. Validation of break-apart and fusion MYC probes using a digital fluorescence in situ hybridization capture and imaging system. J Pathol Inform. 2016;7(1):20. https://doi.org/1 0.4103/2153-3539.181764.

5. Horn H, Bausinger J, Staiger AM, Sohn M, Schmelter C, Gruber K, et al. Numerical and structural genomic aberrations are reliably detectable in tissue microarrays of formalin-fixed paraffin-embedded tumor samples by fluorescence in-situ hybridization. PLoS One. 2014;9(4):e95047. https://doi. org/10.1371/journal.pone.0095047.

6. Magnoli F, Bernasconi B, Vivian $L$, et al. Primary extranodal diffuse large Bcell lymphomas: Many sites, many entities? Clinico-pathological, immunohistochemical and cytogenetic study of 106 cases. Cancer Genet. 2018;228-229:28-40.

7. Rossi G, Jocolle G, Conti A, et al. Detection of ROS1 rearrangement in nonsmall cell lung cancer: current and future perspectives. Lung Cancer. 2017;8: 45-55. https://doi.org/10.2147/LCTT.S120172.

8. Patel NR, Chrisinger JSA, Demicco EG, Sarabia SF, Reuther J, Kumar E, et al. USP6 activation in nodular fasciitis by promoter-swapping gene fusions. Modern pathology. 2017;30(11):1577-88. https://doi.org/10.1038/modpa thol.2017.78.

9. Sugita S, Hasegawa T. Practical use and utility of fluorescence in situ hybridization in the pathological diagnosis of soft tissue and bone tumors. $J$ Orthop Sci. 2017;22(4):601-12. https://doi.org/10.1016/j.jos.2017.02.004.

\section{Publisher's Note}

Springer Nature remains neutral with regard to jurisdictional claims in published maps and institutional affiliations.

Ready to submit your research? Choose BMC and benefit from:

- fast, convenient online submission

- thorough peer review by experienced researchers in your field

- rapid publication on acceptance

- support for research data, including large and complex data types

- gold Open Access which fosters wider collaboration and increased citations

- maximum visibility for your research: over $100 \mathrm{M}$ website views per year

At $\mathrm{BMC}$, research is always in progress.

Learn more biomedcentral.com/submissions 